\title{
Zeitgeschichte und Strafprozessrecht
}

Tom Hayden, führender Exponent der amerikanischen Protestbewegung, nannte kürzlich in einem Interview zwei Morde des Jahres 1968, an Martin Luther King und Robert Kennedy, als ihre Auslöser.

In Deutschland waren es eher zwei Gerichtsverfahren, wobei schwer einzuschätzen ist, wieweit ihre Ergebnisse Folge der Protestbewegung waren oder Ursache. Zwei Freisprüche in spektakulären Prozessen heizten die Stimmung vor allem in Berlin gewaltig auf und sind selbst nur aus der erhitzten Atmosphäre der eingekesselten Stadt zu erklären.

Am 21. November 1967 wurde der Polizeimeister Karl-Heinz Kurras, der am 2. Juni 1967 nahe der Deutschen Oper den Studenten Benno Ohnesorg erschossen hatte, vom Vorwurf der fahrlässigen Tötung freigesprochen, wobei die Moabiter Strafkammer die ungewöhnliche dogmatische Figur des Putativ-Notwehrexzesses entwickelte. ${ }^{1}$ Das Urteil hat das Vertrauen vieler in den Rechtsstaat noch mehr erschüttert als das eigentliche Geschehen, wobei kaum bekannt ist, dass der 5. Strafsenat des Bundesgerichtshofs (BGH) im November 1968 den Freispruch aufhob, aber 1971 wurde Kurras endgültig freigesprochen.

Am 6. Dezember 1968 wurde der ehemalige Richter am Volksgerichtshof und Beisitzer Freislers, Hans-Joachim Rehse, freigesprochen. ${ }^{2}$ Eine Verurteilung Rehses am 5. Juni $1967 \mathrm{zu}$ fünf Jahren Zuchthaus ${ }^{3}$ hatte der BGH am 30. April 1968 aufgehoben. ${ }^{4}$ Die Emotionen, die Rehses Freispruch auslöste, hat der Schriftsteller FriedrichChristian Delius in seinem dokumentarischen Roman „Mein Jahr als Mörder“ hinreißend beschrieben. ${ }^{5}$ So eng der Zusammenhang beider Urteile schon auf den ersten Blick erscheint, bei näherem Hinsehen wird der Zusammenhang noch enger, aber auch verwirrender. Die 14. Große Strafkammer, die so viel juristische Fantasie beim Kurras-Freispruch entwickelte (Vorsitzender: Landgerichtsdirektor Friedrich Geus) war es auch, die den Blutrichter Rehse mit klaren und vernünftigen Erwägungen verurteilte, und der 5. Strafsenat des BGH mit Senatspräsident Werner Sarstedt hob den bizarren Kurras-Freispruch auf, aber auch die plausible Verurteilung Rehses.

Die beiden Freisprüche radikalisierten Teile der Protestbewegung, ${ }^{6}$ und die Strafprozesse gegen die daraus entstandenen Terror-Gruppen „Rote-Armee-Fraktion“ und „Bewegung 2. Juni“ führten zu zahlreichen Änderungen der Strafprozessordnung. Denn einen Reformschub wie die Heinemannsche Strafrechtsreform mit der Entrümpelung des politischen Strafrechts sowie dem Beginn der Reform des Sexualstrafrechts gab

1 Siehe dazu Peter Carstens, Der Fall Ohnesorg. Wendepunkt für Otto Schily, in: Frankfurter Allgemeine Zeitung, Nr. 126 vom 2.6.2007, 8.

2 Das Urteil des LG Berlin ist vollständig abgedruckt bei Jörg Friedrich, Freispruch für die NaziJustiz. Die Urteile gegen NS-Richter seit 1948. Eine Dokumentation, Reinbek 1983, $463 \mathrm{ff}$.

3 DRiZ 45 (1967), 390.

4 BGHSt 9, 302.

5 Friedrich-Christian Delius, Mein Jahr als Mörder, 2. Aufl. Berlin 2004.

6 Vgl. Norbert Frei, 1968 - Jugendrevolte und globaler Protest, München 2008, 126. 
es im Strafprozessrecht nicht. Während im Strafrecht der Anachronismus offen zutage lag und eine Änderung durch andere politische Mehrheiten leicht zu bewerkstelligen war, lag das kompliziertere Strafprozessrecht in den Händen der Ministeriumsexperten, und die bestimmten allein die Richtung, für Politiker weitgehend undurchschaubar.

Die Strafprozessordnung von 1877 war schon ein halbherziger Kompromiss zwischen autoritärem Inquisitionsprozess und reformiertem Anklageprozess gewesen. Karl Binding sprach damals von „Stück- und Flickwerk“ sowie einer „hässlichen Bastardform". Danach haben alle rechtspolitischen Konjunkturen der wechselvollen deutschen Geschichte ihre Spuren in der Strafprozessordnung hinterlassen, die EmmingerReform von 1924, Notverordnungen der Jahre 1931/32 und vor allem die Strafprozessreform 1935, mit der das „Dritte Reich“ nahezu alle rechtsstaatlichen Verbürgungen der Strafprozessordnung geschleift hatte, getreu der von Franz Exner 1933 ausgegebenen Parole: ,anderer Staat - anderer Strafprozess“.

Umso erstaunlicher, dass nach dem Krieg niemand in Deutschland daran interessiert war, den Strafprozess zu entnazifizieren. Man war sich bei Verabschiedung des „Vereinheitlichungsgesetzes“ - die Besatzungsmächte hatten in ihren Zonen Elemente ihrer jeweiligen Rechtsordnung in den Strafprozess implantiert - einig, dass, wie Dallinger im Einklang mit dem damaligen Justizminister Dehler schrieb, ,grundlegende Reformen jedenfalls zur Zeit nicht erstrebenswert" seien. ${ }^{7}$

Die 1950 für spätere Zeiten angekündigte Reform ließ lange auf sich warten, da, wie es in einem Regierungsentwurf hieß, ,übereilte Änderungen sich in verhängnisvoller Weise auswirken und dem Ansehen der deutschen Rechtspflege Abbruch tun würden". ${ }^{8}$ Die einzigen rechtsstaatlichen Errungenschaften des Vereinheitlichungsgesetzes waren das Verbot bestimmter Vernehmungsmethoden in $\S 136$ a StPO, die Streichung der damals als ,typisch nationalsozialistisch“ bezeichneten Haftgründe der Wiederholungsgefahr und der Tatschwere sowie die Wiederherstellung der Gerichtsverfassung von 1877 .

Nach dem Auschwitz-Prozess der Jahre 1963/649 und der ersten großen Verjährungsdebatte des Bundestages im Jahr 1964, ${ }^{10}$ in deren Folge der Beginn der Verjährung von Mord um fünf Jahre verschoben wurde, sah es so aus, als würden die bundesdeutschen Staatsanwaltschaften die Morde der Nazizeit ernsthaft angehen. Man rechnete mit einer ganzen Welle großer NS-Prozesse. Da entschloss man sich im Justizministerium, mit einer, wie es hieß, „kleinen Strafprozessnovelle“11 - in Wirklichkeit war sie viel größer - die Rechte der Verteidigung zu stärken. Die notwendige Verteidigung wurde ausgebaut, ${ }^{12}$ das Akteneinsichtsrecht erweitert und der bis dahin überwachte Verkehr zwischen Verteidiger und inhaftiertem Beschuldigten von allen Beschränkun-

7 Zur Vereinheitlichung des Strafverfahrens: SJZ 5 (1950), 731.

8 BT-Drs. 4/187.

9 Vgl. Hermann Langbein, Der Auschwitz-Prozeß. Eine Dokumentation, 2 Bde., Frankfurt a.M. 2004.

10 Dokumentiert in: Deutscher Bundestag Presse- und Informationszentrum (Hg.), Zur Verjährung nationalsozialistischer Verbrechen. Dokumentation der parlamentarischen Bewältigung des Problems 1960-1979, Teil 1 (= Zur Sache. Themen parlamentarischer Beratung 1980,3), Bonn 1980, $49 \mathrm{ff}$.

11 Strafprozessänderungsgesetz vom 19.12.1964, BGBl. I, 1067.

12 Z.B. in allen Verfahren vor dem Landgericht und nach dreimonatiger Untersuchungshaft. 
gen befreit. Richterliche Vernehmungen wurden parteiöffentlich gemacht, die Richterablehnung erleichtert; Hinweis- und Belehrungspflichten für Polizei und Staatsanwaltschaft eingeführt sowie zwei neue Institute zur Erweiterung des rechtlichen Gehörs geschaffen: Schlussanhörung und Schlussgehör vor Anklageerhebung. Schließlich verbesserte die Novelle sogar das traditionell sehr restriktive Recht der Wiederaufnahme des Verfahrens, indem sie der Staatsanwaltschaft die Möglichkeit nahm, einen die Wiederaufnahme anordnenden Beschluss anzufechten. ${ }^{13}$

So gar nicht in diese Novelle wollte die erst gegen Ende der Beratungen ins Gesetz aufgenommene Ausweitung der Haftgründe passen; der damalige Justizminister Bucher stellte resigniert fest: „Wir sagen, dass wir die Voraussetzungen der Untersuchungshaft enger fassen, genauer umschreiben wollen und müssen feststellen, dass laufend neue Haftgründe geschaffen werden". ${ }^{14}$ Er meinte damit die Wiedereinführung der gerade zehn Jahre zuvor aus dem Gesetz entfernten Haftgründe der Wiederholungsgefahr und der Tatschwere.

Im Zuge der Notstandsgesetze wurde 1968 die Telefonabhörmöglichkeit des $\S 100$ a StPO geschaffen, ${ }^{15}$ die seither in nicht weniger als 18 Gesetzen erweitert wurde.

1968 passierte außerdem ein legislatives Missgeschick; ob es das Verfahrensrecht betraf oder das materielle Strafrecht, war umstritten. Im Bundesjustizministerium hatte man die Verjährungsvorschriften immer dem materiellen Recht zugerechnet und ihre nachträgliche Änderung als Verletzung des Nulla-Poena-Grundsatzes gewertet. Die Herren Dreher, Lackner und Schätzler von der Strafrechtsabteilung sowie Abteilungsleiter Schafheutle hatten alles Erdenkliche vorgebracht, um jede Änderung der Verjährung von NS-Verbrechen zu verhindern. ${ }^{16}$ Dies geschah im Einklang mit der Ministeriumsleitung. Justizminister Ewald Bucher, ein früherer Nazi, ${ }^{17}$ trat daher zurück, als der Bundestag gegen seinen entschiedenen Protest am 13. April 1965 beschlossen hatte, für die Verjährung der Mordtaten des „Dritten Reichs“ die Zeit vom 8. Mai 1945 bis 31. Dezember 1949 ,außer Acht zu lassen“. ${ }^{18}$ Obwohl damit noch keine Änderung der Verjährung verbunden war, denn nach dem damaligen $\S 69$ Abs. 1 StGB ruhte die Verjährung, genauso wie heute nach $\S 78$ Abs. 1 Nr. 2, wenn der Verfolgung ein gesetzliches Hindernis entgegensteht, und ein solches bestand, solange die Strafverfolgung noch von alliierter Genehmigung abhängig war.

Rechtzeitig vor einer absehbaren zweiten Verjährungsdebatte trat am 1. Oktober 1968 das am 10. Mai vom Bundestag einstimmig beschlossene Einführungsgesetz zum Ordnungswidrigkeiten-Gesetz ${ }^{19}$ in Kraft, das so gravierende Auswirkungen auf die Verjährung von Mordbeihilfe haben sollte und in dessen Folge das mit Abstand größte Strafverfahren wegen Mordtaten der Nazi-Zeit eingestellt wurde, das gegen das Personal des Reichssicherheitshauptamts. Am 20. Mai 1969 kassierte der 5. Straf-

$13 \S 372$ Abs. 2 StPO.

14 BT-Prot., 4. Wahlperiode, 132. Sitzung, 6438.

15 Gesetz vom 13.8.1968, BGBl. I, 949.

16 Vgl. die Stellungnahme der Bundesregierung in der 117. Sitzung des 3. Bundestages am 24.5.1960, dokumentiert in: Deutscher Bundestag (wie Anm. 10), 110.

$17 \mathrm{Zu}$ Bucher vgl. Th. Sommer, in: Die Zeit Nr.5/1963 vom 1.2.1963, sowie den Artikel Ewald Bucher, online publiziert auf http://de.wikipedia.org/wiki/Ewald_Bucher (30.3.2009).

18 Gesetz über die Berechnung strafrechtlicher Verjährungsfristen, BGBl. I, 315.

19 BGBl. I, 530. 
senat des BGH ein Urteil des Landgerichts Kiel und stellte das Verfahren wegen Verjährung ein. ${ }^{20}$ Die Staatsanwaltschaft Berlin hatte jahrelang umsonst gearbeitet, elf Staatsanwälte und 23 Spezialisten der Polizei hatten 150.000 Aktenordner ausgewertet und das Material zu drei Hauptkomplexen sortiert: Endlösung, Einsatzgruppen und Massenerschießungen, 2700 Zeugen namhaft gemacht und 18 Verfahren gegen 300 Beschuldigte bis zur Anklagereife vorbereitet, allesamt nur Gehilfen, versteht sich.

Hubert Rottleuthner kleidet seine Feststellung, dass Dreher das gedreht habe, im „Rechtshistorischen Journal“ zwar vornehm in Frageform, macht aber klar: er hat. ${ }^{21}$ Der Entwurf zum EGOWiG war schon 1964 unter Bucher so formuliert worden, und dass dem Spezialisten für Verjährungsfragen die Konsequenzen einer obligatorischen Milderung der Gehilfenstrafe (von lebenslang auf höchstens 15 Jahre, womit sie am 8. Mai 1960 schon verjährt wäre) nicht klar waren, nachdem er selbst in der Anmerkung zu einem BGH-Urteil vom 22. Mai 1962 subtile Erwägungen zu Konsequenzen obligatorischer und fakultativer Strafmilderung auf die Verjährung angestellt hatte, ${ }^{22}$ ist ausgeschlossen. Auch wenn die peinliche Befragung der beteiligten Referenten Dreher, Karl Lackner und Erich Göhler durch Justizminister Ehmke wegen der Panne, die in seine Zeit als Justiz-Staatssekretär fiel, nur zum Ergebnis hatte, dass - so Göhler die Verjährung ,keiner der Herren bedacht“" habe. ${ }^{23}$

Als 1974 klar war, dass es nach diesem Coup trotz der am 4. August 1969 beschlossenen Verlängerung der Verjährungsfrist für Mord von 20 auf 30 Jahre, also bis 1979, dann wurde er für unverjährbar erklärt, zu keiner Lawine von Prozessen wegen Mordtaten der Nazizeit, sondern allenfalls noch zu vereinzelten Verfahren kommen würde, entschloss man sich zu einer Totalrevision des Strafprozessrechts, freilich mit ganz anderer Tendenz als 1964. Das Erste Strafverfahrenreformgesetz (StVRG) sollte der ,erste Schritt auf dem Wege zu einer umfassenden Reform des Strafverfahrensrechts "24 sein. Es beseitigte das gerichtliche Ermittlungsverfahren und übertrug die meisten Befugnisse des Untersuchungsrichters auf die Staatsanwaltschaft. Das führte zu erheblichen Gewichtsverschiebungen, die mit der in Artikel 6 Abs. 3 der Europäischen Menschenrechtskonvention garantierten Waffengleichheit nur schwer vereinbar sind. Außerdem kassierte das Gesetz die meisten der erst zehn Jahre zuvor geschaffenen Verteidigungsmöglichkeiten, insbesondere Schlussanhörung und Schlussgehör.

Auch der für das Frühjahr 1975 anberaumte Prozess gegen Andreas Baader, Ulrike Meinhof, Jan-Carl Raspe und Gudrun Ensslin warf seine Schatten voraus. Dem 1. StVRG, das am 1. Januar 1975 in Kraft treten sollte, wurde kurz vor Weihnachten 1974 eilig ein „Ergänzungsgesetz“25 angefügt, das den Ausschluss des Strafverteidigers aus dem Verfahren vorsah ( $§ 138 \mathrm{a}$ und $\mathrm{b}$ ), die Höchstzahl der Verteidiger auf

20 BGH, NJW 1969, 1181 ff. Zu den näheren Umständen vgl. Marc von Miquel, Ahnden oder amnestieren? Westdeutsche Justiz und Vergangenheitspolitik in den sechziger Jahren (= Beiträge zur Geschichte des 20. Jahrhunderts 1), Göttingen 2004, 339 ff.

21 Hubert Rottleuthner, Hat Dreher gedreht? Über Unverständlichkeit, Unverständnis und Nichtverstehen in Gesetzgebung und Forschung, Rechtshistorisches Journal 20 (2002), 665 ff.

22 NJW 16 (1962), $2209 \mathrm{ff}$.

23 Zitiert nach von Miquel (wie Anm. 20), 222 Fn. 42.

24 BT-Drs. 7/551, 31.

25 Gesetz vom 20. Dezember 1974, BGBl. I, 3686. 
drei (§ 137 Abs. 1 Satz 2) beschränkte und die Verteidigung mehrerer Angeklagter durch denselben Strafverteidiger (\$146) verbot.

In politischen Strafprozessen hatte es in der deutschen Geschichte schon vereinzelt Verteidigerausschlüsse extra legem gegeben. Hans Litten hatte im Felseneck-Prozess nach seinem rechtswidrigen Ausschluss aus dem Saal getragen werden müssen. ${ }^{26}$ Den DDR-Anwalt Friedrich Karl Kaul hatte man wegen angeblich mangelnder Unabhängigkeit in Kommunistenprozessen von der Verteidigung ausgeschlossen (einige Jahre später hob das Bundesverfassungsgericht Kauls Ausschluss auf, wie auch den späteren Otto Schilys aus dem Stammheim-Prozess). Offenbar sah man im Dezember 1974 schon Konfrontationen mit der Verteidigung voraus, jedenfalls wollte man Vorsorge treiben, und tatsächlich wurden mit der neuen Vorschrift schon im Vorfeld der am 21. Mai 1975 eröffneten Hauptverhandlung die Verteidiger Baaders, Croissant, Groenewold und Ströbele, ausgeschlossen. ${ }^{27}$

Auch die anderen beiden Regelungen des Ergänzungsgesetzes hatten mit Stammheim zu tun. Die Bundesanwaltschaft hatte die Anklage arbeitsteilig angelegt: In einzelne Tatkomplexe und deren Rechtsfragen hatte sich jeweils ein Staatsanwalt intensiv eingearbeitet. Um dem Paroli bieten zu können, organisierten sich die Verteidiger genauso und spezialisierten sich entsprechend. Das setzte voraus, dass jeder beteiligte Anwalt eine Vollmacht von jedem Beschuldigten erhielt und jeder Beschuldigte eine Vielzahl von Verteidigern bekam. Diese, nach altem Recht durchaus legitime, Strategie wurde unterbunden, indem einerseits kein Angeklagter mehr als drei Wahlverteidiger haben und andererseits kein Verteidiger mehr als einen Angeklagten vertreten konnte.

Größte Auswirkungen auf das Stammheim-Verfahren und andere Terroristenprozesse hatte der mit dem „Anti-Terror-Gesetz“ vom 18. August $1976^{28}$ neu geschaffene und wegen des Rückwirkungsverbots auf die Taten der Angeklagten gar nicht anwendbare $\S 129$ a StGB (terroristische Vereinigung). Er wurde zum Schlüssel für eine Fülle strafprozessualer Sonderregelungen. Bei einem Verdacht nach $§ 129$ a wurden die Errichtung von Straßenkontrollstellen (§111 StPO) mitsamt der sogenannten Schleppnetzfahndung ( $\$ 163$ d), die Rasterfahndung ( 998 a), die Durchsuchung ganzer Gebäudekomplexe ( $\$ 103$ Abs. 1 Satz 3), die Anordnung von Untersuchungshaft ohne Haftgrund ( $\$ 112$ Abs. 3), der erleichterte Verteidigerausschluss ( 138 a Abs. 2) und die Kontrolle der Verteidigerpost ( $\$ 148$ Abs. 2) möglich.

Am 28. April 1977 wurden nach 192 Verhandlungstagen die Urteile gegen die drei übrig gebliebenen Angeklagten Baader, Ensslin und Raspe verkündet: jeweils drei Mal lebenslänglich. ${ }^{29}$

Mit dem Ende dieses Verfahrens waren weder die Gewalttaten der RAF noch die ständigen Strafprozessrechtsänderungen beendet. Mit der Ermordung des Generalbun-

26 KG, JW 1933, 484.

27 Mit Beschluss vom 3.2.1975 wurde zunächst die Bestellung zu Pflichtverteidigern zurückgenommen. Am 22.4., 2.5. und 13.5.1975 wurden die Verteidiger dann einzeln ausgeschlossen. Vgl. Claus Croissant u.a., Politische Prozesse ohne Verteidigung?, Berlin 1976, $101 \mathrm{f}$.

28 BGBl. I, 2181.

292 StE 1/74. Vgl dazu Martin Schmehl, Die Terroristenverfahren in Stammheim, in: Eberhard Stiltz (Hg.), Das Oberlandesgericht Stuttgart. 125 Jahre. 1879-2004, Villingen-Schwenningen 2004, $63 \mathrm{ff}$. 
desanwalts Siegfried Buback, des Bankiers Jürgen Ponto und des Arbeitgeberpräsidenten Hanns-Martin Schleyer, erreichte der Terror im Herbst 1977 seinen Höhepunkt und setzte sich in den 80er Jahren mit der Ermordung des Siemens-Managers Karl Heinz Beckurts, des Diplomaten Gerold von Braunmühl (1986), des Vorstandssprechers der Deutschen Bank Alfred Herrhausen (1989) und des Treuhandchefs Detlev Karsten Rohwedder (1991) bis zur Auflösung der RAF im Jahr 1992 fort.

Im „,deutschen Herbst“ des Jahres 1977, während der Entführung des Arbeitgeberpräsidenten Schleyer, verhängte die Regierung eine Kontaktsperre über die einsitzenden RAF-Mitglieder, die insbesondere gegen die Verteidiger wirkte, indem sie ihnen jeden Kontakt mit den einsitzenden Mandanten unmöglich machte. Da dieser Grundrechtseingriff keine gesetzliche Grundlage hatte, beschlossen Bundestag und Bundesrat binnen zweier Tage das Kontaktsperre-Gesetz vom 30. September 1977, ${ }^{30}$ das nach Uwe Wesel „schnellste Gesetz der neueren Rechtsgeschichte“. ${ }^{31}$ Man vergisst heute leicht, dass es noch immer in Kraft ist, da es an unscheinbarer Stelle platziert ${ }^{32}$ und seit seinem Inkrafttreten vor 30 Jahren nie angewandt wurde. Zwar gab es die erwähnte ungesetzliche Kontaktsperre, aber seit Geltung des Gesetzes keine mehr.

Diese Geschwindigkeit des Gesetzgebers wurde üblich bei den sogenannten „AntiTerror-Gesetzen“: Am 1. Juni 1978 hatte sich Bundeskanzler Schmidt gegen eine „uferlose Gesetzgebung“ zur Bekämpfung des Terrorismus ausgesprochen, erklärt, dass man keine neuen Gesetze brauche und sich gegen die „ewigen Basteleien“ am Strafprozessrecht ausgesprochen. ${ }^{33}$ Eine Woche später, am 8. Juni 1978, spät abends, lag dem Bundestag ein neues „Anti-Terror-Paket“ vor. Das Bundestagsprotokoll verzeichnet dazu folgende Worte der Vizepräsidentin Renger: „Wir stimmen jetzt über Artikel $1 \mathrm{Nr} .13$ in der Ausschussfassung ab. Wer dem zuzustimmen wünscht, den bitte ich um das Handzeichen. - Gegenprobe! - Enthaltungen? Einstimmig so angenommen. Ich rufe nunmehr Punkt 1 Nr. 14-34 und Punkt 2-9 in der Ausschussfassung sowie Einleitung und Überschrift auf. Wer dem zuzustimmen wünscht, den bitte ich um ein Handzeichen. - Gegenprobe! - Enthaltungen? - Einstimmig so beschlossen, in zweiter Beratung angenommen. Wir treten nunmehr in die dritte Beratung ein. Wird dazu noch einmal das Wort gewünscht? - Das ist nicht der Fall. Wir kommen zur Schlussabstimmung. Wer dem Gesetz als Ganzen zuzustimmen wünscht, den bitte ich, sich zu erheben. - Gegenprobe! - Enthaltungen? Das Gesetz ist einstimmig so angenommen". 34

Der Bundestag hatte damit binnen zweier Minuten, in zweiter und dritter Lesung, das sechste strafprozessändernde Gesetz seit 1975 beschlossen und wie seine Vorläufer brachte es neue Beschränkungen der Verteidigung. Es strich kurzerhand ihre Befugnis, mit der Vorlage von Beweismitteln deren Verwertung zu erzwingen. Nach dem neuen $\S 245$ StPO ist die Beweisaufnahme ,auf alle vom Gericht vorgeladenen und auch erschienenen Zeugen und Sachverständigen sowie auf die sonstigen ... vom Gericht oder der Staatsanwaltschaft [also nicht von der Verteidigung] herbeigeschafften Beweismittel zu erstrecken“; ein offenbarer Verstoß gegen Art. 6 Abs. 3 d der Eu-

30 BGB1. I, 1877.

31 Die Zeit, Nr. 43 vom 22.10.1997.

32 In den $\S \S 31$ bis 38 a EGGVG.

33 Vgl. Ingo Müller, Vorgänge 34/1978, 14.

34 BT-Prot., 8. Wahlperiode, 7550. 
ropäischen Menschenrechtskonvention, die jedem Angeklagten das Recht garantiert, „die Ladung und Vernehmung der Entlastungszeugen unter denselben Bedingungen wie die der Belastungszeugen" zu bewirken. Außerdem wurden nicht nur die Erleichterungen der Richterablehnung aus der Strafprozessnovelle 1964 beseitigt, sondern die Ablehnung des befangenen Richters zusätzlich erschwert. Mit dem abgelehnten Richter muss weiter verhandelt werden, bis eine Entscheidung „ohne Verzögerung der Hauptverhandlung“ möglich ist. Weiterhin führte das Gesetz die „,qualifizierte Rügepräklusion" ein. Der Einwand, das Gericht sei vorschriftswidrig besetzt, kann nur bis zum Beginn der Vernehmung des ersten Angeklagten in der Hauptverhandlung geltend gemacht werden, wobei auch die Tatsachen, aus denen sich die falsche Besetzung ergibt, anzugeben sind. Wird eine falsche Besetzung nicht gerügt, kann auch die Revision nicht auf sie gestützt werden. Das neue Ablehnungsrecht sollte Missbräuchen, wie sie angeblich im Stammheim-Prozess vorgekommen seien, künftig vorbeugen. Dabei hatte gerade dort erst die 87. Ablehnung, ausgerechnet durch einen der berüchtigten ,Zwangsverteidiger“, erwiesen, wie befangen der Vorsitzende Richter Prinzing tatsächlich war. Darin sahen die Gesetzesbegründer des Justizministeriums aber, dass „das für die Funktion der Justiz höchst bedeutsame Institut der Richterablehnung wegen Besorgnis der Befangenheit eine missbräuchliche Handhabung" ermögliche.

Mit den ständigen Änderungen von Gerichtsverfassungsgesetz und Strafprozessordnung hatte das deutsche Strafverfahren schließlich einen Zustand erreicht, der sich sogar zum Vorwand für Abschiebungen eignete. Dem langjährigen Vorsitzenden der Rechtsanwaltskammer des türkischen Bezirks Mus, Serafettim Kaya, der nach Verteidigungen vor einem türkischen Militärgericht selbst inhaftiert und gefoltert wurde und dem eine Anklage vor einem solchen Gericht bevorstand, verweigerte, wie die Frankfurter Rundschau am 9. Dezember 1982 berichtete, ${ }^{35}$ das Bundesamt für die Anerkennung ausländischer Flüchtlinge das Asyl mit der bemerkenswerten Begründung: „Dem Bundesamt liegt ein umfangreiches Gutachten des Max-Planck-Institutes vor, das unter anderem auch eine synoptische Gegenüberstellung des türkischen Militärstrafverfahrensrechts mit der deutschen Strafprozessordnung enthält. Dieser Vergleich zeigt eine weitgehende Übereinstimmung, teilweise sogar eine liberalere Ausgestaltung des türkischen Militärverfahrensrechts ...".

Welchen Anteil die Terroristen-Verfahren an der Strafprozessnovellen-Konjunktur der 70er Jahre tatsächlich hatten, lässt sich aber schwer einschätzen. Seit dem Ersten Weltkrieg hatten stets echte oder angebliche Notstände als Vorwände für Strafprozessänderungen herhalten müssen. Mag eine zu bewältigende Krise auf Inflation, Staatsstreiche (1923/24), drohenden Staatsbankrott (1930), totalen Krieg (1942/44), Bedrohung des Staates durch Terrorismus (1975/78) oder überlange Dauer des Strafverfahrens (1979) zurückzuführen sein, die Vorschläge der Justizbürokratie waren immer dieselben. Als zum Beispiel im Jahr 1923, dem turbulentesten der krisengeschüttelten Weimarer Republik, alles drunter und drüber ging - die Inflation galoppierte, Franzosen besetzten das Ruhrgebiet, die Reichswehr marschierte in Sachsen ein und Hitler putschte in München - erhielt die Regierung umfangreiche Notbefugnisse zur Bewältigung der Krise und vier Wochen später erging eine Notverordnung, welche die Rechtsmittel in Strafsachen reduzierte, das Beweisrecht der Verteidigung beschnitt und vor 
allem die populären, aber in der Justizbürokratie verhassten Schwurgerichte abschaffte. ${ }^{36}$

Der eifrigste Protagonist der Strafprozessänderungen der 70er und auch 80er Jahre hatte noch viel weitergehende Pläne: ${ }^{37}$ In Strafrichter-Sachen - über 90 \% aller Strafprozesse - ein vereinfachtes Verfahren ohne Beweisantragsrecht, Verwertung polizeilicher Vernehmungen im Hauptverfahren und Beseitigung des Richtervorbehalts bei allen Grundrechtseingriffen. Dazu ist es bekanntlich nicht gekommen. Sei es, dass Hölderlin Recht hat und, wo die Gefahr am größten, das Rettende tatsächlich nachwächst, sei es, dass 1968 und die Folgezeit für alle Beteiligten ein großer Lernprozess war.

Festzustellen ist, dass trotz eklatanter Verschlechterungen der Strafprozessordnung sich die Möglichkeiten der Strafverteidigung seither erheblich verbessert haben. Die Lehren von Stammheim und der Kampf gegen die ständige Verschlechterung ihrer prozessualen Möglichkeiten haben einen Professionalisierungsschub bei deutschen Strafverteidigern ausgelöst. Sie gründeten eine inzwischen angesehene Strafprozess-Zeitschrift, veranstalten Strafverteidigertage, übernahmen die Meinungsführerschaft in Strafprozessfragen und setzen den Fachanwalt für Strafrecht durch. Die einst als Linksanwälte Beschimpften mutierten zu hoch angesehenen Strafverteidigern. Kein bedeutendes Wirtschaftsstrafverfahren ohne einen aus diesem Kreise. Einer wurde Justizminister in Hessen, ein anderer gar Bundesinnenminister und manche Sottise des Stammheim-Prozesses, wie Rupert von Plottnitz’ „Heil, Dr. Prinzing“ oder Otto Schilys „Ihre Robe wird immer kürzer und das Krokodil darunter immer sichtbarer", haben sie sicher oft bereut. Der damalige Justizminister Vogel mag Recht haben mit seinem Wort, dass sich beim Marsch durch die Institutionen diese sich weniger verändert hätten als die Marschierer, aber das musste nicht immer ein Nachteil sein. Denn auch die Richterschaft hat sich, wie Dieter Simon beschreibt, gewandelt: „Eine neue Generation von Richtern trat in Erscheinung“, und mit ihnen sowie der Pensionierung der letzten alten hielt ein neuer Ton Einzug in die Gerichtssäle, breitete sich „eine erfreuliche Sensibilität aus““.38 Inzwischen begegnet man einander sogar im Strafprozess mit mehr Respekt und schöpft die prozessualen Repressionsmöglichkeiten des Gesetzes bei weitem nicht aus. Claus Roxin schreibt im Vorwort zur populären dtv-Ausgabe der StPO, dem Strafprozessrecht komme ,symptomatische Bedeutung für den Geist einer Rechtsordnung zu“. ${ }^{39}$ Bei dem Zustand, den die StPO in den letzten 30 Jahren erreicht hat, wäre das ein beängstigender Gedanke; glücklicherweise ist es nicht so. Die Strafjustiz ist mittlerweile rechtsstaatlicher als unsere verhunzte Strafprozessordnung.

36 Die sogenannte Emminger-Verordnung vom 4.1.1924, RGBl. I, 15.

37 Peter Rieß, Prolegomena zu einer Gesamtreform des Strafverfahrensrechts, in: Helwig Hassenpflug (Hg.), Festschrift für Karl Schäfer zum 80. Geburtstag am 11. Dezember 1979, Berlin u.a. $1980,155 \mathrm{ff}$.

38 Dieter Simon, Zäsuren im Rechtsdenken, in: Martin Broszat (Hg.), Zäsuren nach 1945. Essays zur Periodisierung der deutschen Nachkriegsgeschichte (= Schriftenreihe der Vierteljahrshefte für Zeitgeschichte 61), München 1990, 164.

39 Strafprozessordnung mit Auszügen aus Gerichtsverfassungsgesetz, EGGVG, Jugendgerichtsgesetz, Straßenverkehrsgesetz und Grundgesetz. Textausgabe mit ausführlichem Sachregister und einer Einführung von Universitätsprofessor Dr. Dr. h.c. mult. Claus Roxin, 44. Aufl. München 2008, X. 
Die Justiz ist nämlich recht pluralistisch und, wie die Gesellschaft insgesamt, demokratischer geworden. Die Träume des ersten BGH-Präsidenten, Hermann Weinkauff, der - nicht zufällig gerade 1968 - „die pluralistische Gesellschaft“ sowie den „Pluralismus der Weltanschauungen“ „Dinge“ nannte, ,bei denen die Sache so bedrohlich, wie die Bezeichnung widerwärtig ist,“ und der damals noch von einem ,geschlossenen Richterstand, ja einem wirklichen Rechtsstand“40 schwärmte, haben sich gottlob nicht erfüllt. Das ist wohl die nachhaltigste Errungenschaft der Juristen nach 1968.

40 Hermann Weinkauff, Die deutsche Justiz und der Nationalsozialismus. Ein Überblick, Bd. 1 (= Quellen und Darstellungen zur Zeitgeschichte 16,1), Stuttgart 1968, 180, 188. 